\title{
Construcción de la educación mediática por comunidades de aprendizaje horizontales.
}

\author{
Raúl ANTÓN CUADRADO \\ Licenciado en Informática - Colaborador docente, UNED \\ raulanton@gmail.com \\ Valeria LeVRATTO \\ Licenciada en Ciencia de la Comunicación (Turín, Italia)- Colaborador docente, UNED \\ valevratto@gmail.com
}

\begin{abstract}
Resumen:
En esta comunicación se pretende recrear el escenario pedagógico de la edición de 2013 de la asignatura Didáctica de la Educomunicación (Facultad de Educación, UNED).

En ella, los entornos virtuales, se utilizaron desde planteamientos constructivistas y horizontales, con el conectivismo y la teoría del caos como catalizadores haciendo al alumno sujeto del aprendizaje.

Ellos elaboraron el itinerario participando en la comunidad de aprendizaje, decidieron en torno a qué actividades problematizar y consensuaron el método de evaluación de los docentes. Sus aportaciones críticas, se integran en aquilatan en mejores prácticas y focos de mejora.
\end{abstract}

Palabras clave: Comunicación horizontal - Didáctica de la Educomunicción - Comunidades de aprendizaje - Constructivismo - Conectivismo

\section{Building Media Literacy trough horizontal learning communities}

\begin{abstract}
:
The aim of this paper is to present the pedagogical scenario developed in 2013 Media Literacy Pedagogy lectures. (Education, UNED).

Virtual environments have been used through horizontal and constructivist proposals, using connectivism and chaos theory as catalyzers, to make students active subject of learning process.

They generated the itinerary, participating in a community and choosing the discussion areas. They also agreed on the evaluation method. Their analsyis of the experience done have been collected to create best practices and improvement opportunities.
\end{abstract}

Key Words: Horizontal communication - Media Literacy didactic - Learning communities - Constructivism - Conectivism

\section{Referencia normalizada:}

Antón Cuadrado, R. y Levratto, V. (2013) Construcción de la educación mediática por comunidades de aprendizaje horizontales. Historia y Comunicación Social. Vol. 18 No Especial Octubre. Págs. 231-241

Sumario: 1. Introducción. Estado de la cuestión. 2. Plan didáctico de la experiencia. 2.1. Compromiso con el constructivismo. 2.2. Catalizando la experiencia. Conectivismo, caos, autopoiesis. 2.3. Entornos Virtuales. 3. Narración etnográfica y grupo de discusión. 4. Una experiencia de la que aprendemos todxs. 5. Evaluación de la experiencia. 6. Conclusiones. 6.1 Mejoras futuras. 6.2 Reciclaje de la experiencia formativa 7 . Referencias bibliográficas. 


\section{Introducción. Estado de la cuestión}

\footnotetext{
"¿Alguna vez habéis sentido que algo empezaba cuando estaba terminando? ¿Alguna vez os habéis sentido junt@s en la distancia? ¿Alguna vez creísteis conocer a alguien cuando jamás le habiais visto?" (Victor, Alumno)
}

Bourdieu y Passeron percibieron que el sistema de enseñanza viene sirviendo a la reproducción de los esquemas sociales y las relaciones de poder que los sustentan (1979) Nada que contenga una emancipación. Más bien un adoctrinamiento en el que es inadmisible un resultado no esperado del sistema, porque se busca la homogeneización -en el sentido más industrial- del resultado. Para ello, se busca eliminar las variables complejas, caóticas, entre ellas la participación de los aprendices salvo para dar un feedback de asimilación de la narración unidireccional en el que la curiosidad y el conocimiento languidecen. "Cuando más analizamos las relaciones educador-educandos dominantes [...] más nos convencemos de que estas relaciones presentan un carácter especial y determinante, el de ser narraciones de naturaleza fundamentalmente narrativa, discursiva, disertadora" (Freire, 1970: 51). Nuestra experiencia educativa busca la emancipación no con el objeto de integrar a los aprendices en la estructura, sino con el de permitirles transformarlas, empezando por la horizontalización de las asfixiantes relaciones de poder en los ámbitos educativos, superando "la contradicción educador-educando [...] de tal manera que ambos se hagan, simultáneamente, educadores y educandos" (ibíd, 77).

\section{Plan didáctico de la experiencia}

La educación ha consistido muy frecuentemente en "la transmisión de conocimientos y valores de una generación a otra, del profesor al alumno, de la élite instruida a las masas ignorantes" (Kaplún, 1998: 18), "para ser memorizados y aprendidos por los educandos" (Kaplún, 1997: 1). Este diseño desmantela el desarrollo crítico del alumno, pero también daña al educador que renuncia a aprender de un proceso horizontal y no añade nada respecto a un medio de broadcasting, siendo cuestión de tiempo evolucionar el 'magister dixit' hasta el 'anunciado en televisión' o el 'lo pone en la wikipedia': tres versiones de la perezosa renuncia a interrogarse críticamente.

El sistema convierte a los alumnos en objetos, "la narración los transforma [a los educandos] en vasijas, en recipientes que deben ser llenados" (Freire, 1970: 51). La horizontalidad les recupera como sujetos de sus propios procesos de aprendizaje, preservando su curiosidad como "motor esencial del conocimiento" (Freire, 2003: 21). La emancipación del argumento de autoridad, de la historia única (Adichie, 2009) es inevitable con el acceso al conocimiento global que Internet propone. Pero exige de un esfuerzo, re-ilustrarse, asumir la mayoría de edad. Enseñante y aprendiz deben tomar posición para ganar su libertad. 


\subsection{Compromiso con el constructivismo}

Muscular el aparato crítico del aprendiz no es compatible con inculcar "nuevas actitudes sin pasar por la reflexión, por el análisis" (Kaplún, 1998: 35). No se trata de asegurar la repetitividad del resultado, sino que declinando "referirse a la realidad como algo detenido, estático" (Freire, 1970: 75), se privilegia el aprendizaje por experimentación, dislocando el fenómeno del aprendizaje, inscribiendo el proceso en el horizonte vivencial del alumnado, haciéndole sujeto. Así, se ha fomentado el conflicto que Kaplún preconizaba como generador de conciencia crítica, por medio de su socialización en una discusión participativa que ha tenido lugar en los foros, entre otras herramientas virtuales(1998).

El docente, liberado de la repetición del mismo credo, se convierte en "mediador de experiencias" (Silva, en Aparici 2009: 9), en acompañante, en animador de la dinámica. No renuncia a aportar información para que el grupo avance, como admite Freire, pero sometiendo ésta al espíritu crítico de los alumnos. Concebimos el diálogo maestro-educandos horizontal, como práctica de libertad de ambos en el marco de la comunidad de aprendizaje. Pero esto añade nuevas variables en el proceso de construcción de conocimiento que lo hacen caótico, no determinista. No se puede anticipar el resultado en cada alumno, algo esencial en otros paradigmas. Aceptamos conectivismo y caos, ¿Cuáles son sus implicaciones?

\subsection{Catalizando la experiencia. Conectivismo, caos, autopoiesis}

Caos no es desorden. "El caos señala que el significado existe, y que el reto del aprendiz es reconocer los patrones que parecen estar escondidos. La construcción del significado y la formación de conexiones entre comunidades especializadas son actividades importantes." (Siemens, 2004: 5). En la asignatura, no se plantea individualmente el desentrañamiento de estos patrones, sino desde los principios del conectivismo, por la participación en comunidades de aprendizaje que se erigen en suprafenómenos autopoiéticos.

La autopoiesis define el acoplamiento del sistema a su entorno y es la característica de los sistemas que lo que fabrican es a ellos mismos. Los seres vivos son sistemas autopoiéticos [...] la muerte es precisamente la interrupción de esta modificación continua que preserva al sistema frente al medio (Antón, 2010)

En la autopoiesis, neologismo de Maturana (1970) a partir del griego aútós (autós),

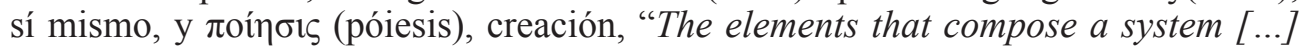
are its components only as they participate in its composition and only while they do so." (Maturana 2002: 30). Así, la comunidad existe como ente y los alumnos se benefician de un aprendizaje extendido formando una estrecha red de relaciones con sus compañeros, participando en ella. La construcción de la propuesta didáctica partió de la confianza en la autonomía y autoregulación de esta comunidad de aprendizaje que afronta las perturbaciones, adaptándose, formando nudos de inteligencias mutuamente alimentadas, sabiendo que "el aprendizaje es un proceso que ocurre en 
el interior de ambientes difusos de elementos centrales cambiantes - que no están por completo bajo control del individuo" (Siemens, 2004: 7).

La comunidad de aprendizaje y el cerebro del individuo no son sino ambientes fractales, escenarios de aprendizaje replicados a distinto nivel. Ramón y Cajal lo anticipó hace más de un siglo y los teóricos del conectivismo reconfirman sus planteamientos "The brain as a whole operates more like a social network than a digital computer" (Downes, 2005). La fisiología humana anima la necesidad de relación tomando parte en tejidos reticulares y las TIC sobre la red ofrecen un panorama ideal sobre el que conectarse en una "inteligencia colectiva" (Levy, 2006).

\subsection{Entornos virtuales}

La elección de los EVI determina una narrativa y modelo de interrelación y, como recuerda Osuna, "éstos deben de facilitar la enseñanza como proceso de apoyo, trascendiendo la comunicación unidireccional de conocimientos" (2007: 86). Deben de fomentar la participación horizontal, autónoma y crítica, sin recelar de lo externo a la institución, celebrando aprendizajes cuando sea posible, con experiencias en la propia Red.

Amén de medios en Internet, se buscó un eje-indice para vertebrar la comunidad en la plataforma de la UNED. Los blogs reifican una relación de poder que segrega al autor del post y de los comentarios. Las wiki focalizan en la historia única de consenso más que el proceso de aprendizaje. Así, se hizo de los foros el eje de la construcción de aprendizaje, ritmándolos con chats -facultativos y cuyo contenido quedaba grabado y accesible- que proponían revisiones críticas de las lecturas y la socialización del avance en las actividades acordadas. Los foros permiten:

La motivación hacia la construcción activa y social del conocimiento, el aumento de las posibilidades de comunicación, mayor trabajo autónomo, facilidad para la discusión grupal, mejora en la argumentación de las ideas, potencia la comunicación escrita, mejora la capacidad de síntesis, de negociación... (Osuna 2007: 31).

Los entornos virtuales se escogieron para buscar la horizontalidad de la participación y la generación autopoiética de la comunidad. Según los alumnos, se consiguió.

"de esta asignatura me ha gustado lo poco directiva que ha parecido" Francisco.

"tienen el rol de orientadores, de facilitadores, no es el modelo directivo el que ustedes han seguido, sino el de acompañar desde al lado" Susana Regina.

"a mi me ha gustado poder decidir entre todos qué será evaluado y cómo" Walter.

"[el rol de los enseñantes ha sido] poner hitos en el camino y animarnos con vuestro entusiasmo" Samantha. Javier.

"habéis permitido que sobre vuestro vehículo, nosotros hayamos conducido"

“dejáis espacio para que nosotros también podamos proponer" Alicia. 


\section{Narración etnográfica y grupo de discusión}

La metodología de recolección de datos para la validación de la experiencia, se ha construido sobre la observación participante que ha permitido el acceso al discurso generado, especialmente en los foros, y la celebración de un grupo de discusión de los alumnos, para "conocer y explicar cómo percibe la gente una determinada realidad, qué piensa y qué siente ante ella". (Gil Flores, 1992-1993: 202). Observación participante y entrevista -o en nuestro caso el grupo de discusión, más rico al integrar todas las voces en un único discurso- se complementan ya que "la observación participante proporciona [...] discurso propio del investigador; la entrevista, [...] proporciona discurso ajeno, de los sujetos de estudio" (Díaz de Rada y Velasco 1996: 34)

La presentación de la experiencia se realiza en forma de narración etnográfica con el propósito de reconstruir el contexto, dar "una pintura holística" (Genzuk, 2003) sobre la cual cobran significado los resultados de la investigación, como un modo "de dar coherencia a nuestros datos en relación con nuestra experiencia de campo" (Ardevol, 2010).

\section{Una experiencia de la que aprendemos todxs}

La guía didáctica de la asignatura así como el apartado de "actividades" han predeterminado que la comunidad de aprendizaje era la que iba a ser coautora de contenidos de la misma manera que iba a acordar, de manera consensuada, la ponderación de las actividades sugeridas. Ambas actividades generaron entusiasmo y motivación entre el alumnado; sobre todo a nivel de aprendizaje el equipo docente favoreció la creación de espacios virtuales abiertos donde todos y todas pudiesen tener voz y dejar su huella de experiencia y construcción del conocimiento. Los foros han tenido un papel privilegiado seguido por las distintas herramientas comunicativas que la Web ofrece.

A nivel de evaluación el análisis de experiencias educomunicativas ocupaba un espacio considerable en los equilibrios finales de la asignatura; a través de una descripción ensayista de las realidades encontradas en Internet o gracias a experiencias personales, el alumnado ha podido reflexionar críticamente sobre ellas juntando así estudios teóricos con casos prácticos y originales.

En un enfoque constructivista el equipo docente no pudo que simplemente marcar unas pocas líneas y pautas para dar empiezo a la discusión que era la que finalmente hubiera guiado el camino de los alumnos en sus ensayos personales.

El entusiasmo de la comunidad de aprendizaje no se simplifica si se reproduce una única voz altamente representativa: "Nunca hasta ahora había sido partícipe de la horizontalidad que supone el hecho de discutir y consensuar las actividades y su ponderación entre todos los miembros de una comunidad de aprendizaje-docentes 
y alumnado" (Javier) De dicha consensuación, no fácil sin duda, salió una serie de preguntas comunes para que todos se moviesen gracias y con ellas en su análisis.

Una vez más a través de un foro se han ido compartiendo experiencias educomunicativas de lo más distinto y interesante, entre ellas 'Media Literacy Project' de Nuevo México o el colombiano 'Prensa-Escuela' al 'Hole in the wall', una experiencia de educación a través de ordenadores en India. En este proceso se ha ido perfilando un mapa mental mundial donde cada estudiante ha podido investigar y bucear para dar una consistencia más global e intercultural a la educomunicación.

La segunda actividad objeto de evaluación de la asignatura surgió gracias a la espontaneidad de, respectivamente, Andrés y Judith: "El acto formativo no sólo reside en las instituciones educativas como espacio formales y oficiales del conocimiento, sino fuera de las instituciones, en la educación no formal, informal y la familia, los padres". A veces olvidados, "los padres son un colectivo, que a mi parecer también debería formarse". Sin darse cuenta, estas simples afirmaciones movieron un amplio debate que marcó el escenario de la actividad para realizar colaborativamente.

El tema de la educomunicación a padres de alumnos fue el objeto de un animado y intenso dialogo que día a día crecía en los foros donde el equipo docente, una vez más, sólo estimulaba y animaba la discusión pero donde los alumnos fueron "libres para crear cuantos hilos consideren oportunos para categorizar y vertebrar la reflexión" (Acuerdo sobre actividades).

De todo ello se citan a modo ejemplifico cinco interesantes debates entre las más prolíficas:

- ¿Estamos preparados para el coaprendizaje?

- ¿Qué es exactamente la identidad digital?

- ¿La escuela educomunicadora debe formar a las familias? Este punto en particular es la prueba del nueve de nuestra experiencia de aprendizaje, ya que cuestionaba la propia viabilidad y oportunidad de la actividad.

- ¿Cómo utilizar los videojuegos como recurso de educación?

- ¿Qué herramientas en la red deben conocer los padres?

Kaplún (1998) nos enseña que principio clave de cualquier educomunicador es el de tener una actitud creativa a la vez de una recepción critica, ambas indispensables, por lo tanto a lo largo de la asignatura se animó a la creación, a partir de las fértiles discusiones que se habían desarrollado en los foros.

Con mucha motivación, creatividad y pensamiento critico se crearon unas píldoras así denominadas por su breve duración; en realidad la del tiempo fue la única restricción que el equipo docente propuso a la comunidad. El carácter audiovisual era imprescindible para poderlas compartir en los escenarios virtuales, estas creaciones iban juntas a un manual de uso de formato igualmente libre, conteniendo una "propuesta de ejercicio participativo en torno al material presentado [...] sin descartar actividades de tipo intergeneracional, primando las de carácter lúdico" (Acuerdo sobre actividades). 
De todo ello han nacido cinco vídeos utilizables bajo licencia $\mathrm{CC}$ que se pueden ver en la página:www.comunicacionextendida.com.

\section{Evaluación de la experiencia}

La sinergia del tod@s aprendemos con tod@s acompañó incluso el momento de evaluación de la experiencia cuando el equipo docente, terminado el periodo electivo y de calificación propuso un grupo de discusión virtual, que un alumno definió "off the record y parte del espiritu global"; además animó el alumnado a crear un anexo a la entrega de los informes de las actividades.

Conscientes de que la motivación es precursora en un proceso de aprendizaje, los profesores habían estructurado la actividad de la píldora audiovisual de manera tal de poder crear un objetivo colectivo que fuese más allá de la simple nota de la asignatura del Máster. Las creaciones serán parte de un proyecto común del profesorado que podrá utilizarlas como interesante y practico material para ponencia relacionadas al tema.

Por esa razón a final del curso un alumno comentó:"la sensación de gratificación es aún mayor cuando lo que la produce es un proceso y no un resultado"haciendo referencia a la importancia de sentirse parte de un todo que no empieza y termina con los tiempos académicos sino que va, al menos potencialmente, más allá.

Cuando llegó el momento de evaluar de forma colectiva la experiencia se eligió un focus group para la recolección de datos. Los docentes fueron los mediadores de este proceso procurando aproximarse al principio básico que sugieren algunos expertos en metodología que"el entrevistador sea un hábil conversador y que sepa llegar a los aspectos más profundos de las personas".(Hernández, R., Fernández, C., Baptista, P., 2006: 473).

El caos creativo que animó la dinámica de las clases emergió entre los puntos más relevantes del debate:"Sentirse perdido es bueno para avanzar[...] Si solo pretende seguir como estaba, es mejor una propuesta tradicional, que no supone demasiados retos (salvo memorizar)" (Javier), "ese proceso de adaptación te hace crecer y valorar esta otra forma de aprender" (Samantha) y junto con ello se apreció un proceso que fuese cuanto más personalizado:"siento que ha estado hecha a mi medida, a la medida de cada uno de nosotros" (Javier).

La satisfacción y aprendizaje interesante se reflejan en las palabras de un alumno y una alumna que muy bien expresan estos momentos:"Me ha gustado poder decidir entre todos que será evaluado y como, esta cosa más democrática has sido novedosa"; "Creo que la propuesta de las actividades y la evaluación guardó absoluta sintonía con los contenidos, el trabajo grupal fue muy interesante porque resultó realmente un trabajo colaborativo, nada directivo y absolutamente autogestido en el grupo.". 
De todo ello no podían que salir algunas palabras claves y entre las más repetidas cuando los alumnos han debido describir, de la forma más espontanea posible, la experiencia global de la asignatura: coautoría, debate, coaprendizaje, autonomía, mediación, autogestión, definitivamente todas imprescindibles en el glosario de una didáctica resueltamente horizontal.

\section{Conclusiones}

A continuación se quieren destacar algunos puntos que resultan esclarecedores de una metodología utilizada que ha determinado que la experiencia de la clase se pueda considerar por lo general satisfactoria:

- En un ámbito educomunicativo la creación es uno de los pilares fundamentales entornos a los cuales es preciso moverse y en este caso se ha demostrado clave para poder entender los distintos momentos de aprendizaje. La experiencia, como nos enseñó el maestro americano Dewey es la que genera el pensamiento y además se constituye como la base del aprendizaje.

- Mantener viva la motivación con el estimulo de un proyecto común es algo que ha hecho que la creación colaborativa de píldoras audiovisuales fuese muy estimulante, pudiendo ir más allá del mero proceso de evaluación sino situándose en un momento personal de construcción personal de conocimiento.

- En la Web 2.0 ceñirse al uso del texto escrito para las creaciones tantos colectivas como individuales no sólo sería erróneo sino que totalmente anacrónico y así se ha demostrado en la asignatura donde el equilibrio entre material audiovisual y textual ha permitido perfeccionar el uso y el conocimiento sobre las herramientas que nos ofrece la Web.

\subsection{Mejoras futuras}

Si bien, como se ha comentado, las criticas positivas hayan sido notables y muy enriquecedoras ya que los aprendizajes generados vienen vistos como "más profundos y duraderos y además motivadores" (Samantha) y en definitiva "Mejores porque desarrollan nuestra competencia comunicativa, de trabajo en equipo, de crítica y autocrítica...” (Alicia). "Experimentándolo, construyéndolo, compartiéndolo y exponiéndolo", se aprende "con volumen" (Samantha) existen criticas que son imprescindibles para seguir evolucionando en la asignatura.

Entre las cosas evocadas como "problemáticas" se releva la desorientación y inseguridad experimentada en las primeras fases de estudio, debido a una metodología muy distinta a lo convencional y a las responsabilidades en el diseño general del proceso de enseñanza-aprendizaje en el cual el alumnado se vio involucrado. Por lo tanto, lo que se propuso fue que se implementara una estrategia de acompañamiento para que se pudieran reducir al mínimo estas sensaciones. 
El eventual uso de una red social como Facebook se consideró como una posible estrategia, por adoptar, debido a que en esta plataforma se pueden crear grupos donde profesores y alumnos se ayudan, avanzando y resolviendo dudas, fortaleciendo la sensación de comunidad y generando seguridad y sentimiento de escucha; además es un excelente feedback informal para que el profesorado se pueda hacer una idea general de la situación.

Otro punto interesante que nació de la discusión fue el de las lecturas recomendadas cuya modalidad, totalmente libre y orientativa, se entendió sólo al final; por lo tanto, algunos alumnos y alumnas comentaron la importancia de "hacernos entender, en primer lugar, que las lecturas son propuestas variadas, a las que tenemos que acercarnos de forma no tradicional (leer todo, memorizar) [...] que son diferentes caminos, que pueden recorrerse en diferentes momentos de forma no lineal" (Javier).

La posibilidad de compartir las experiencias y creaciones de los compañeros es algo que ha generado sentimientos muy positivos y que cuando no viene a ser posible se echa en falta. Se llega a reclamar lo positivo que hubiese sido compartir "una especie de abstract"'(Alicia) de los análisis de experiencias educomunicativas de los compañeros y además de poder aprovechar de la lectura de las píldoras de los compañeros lo antes posible.

De la misma manera, el alumnado ha echado en falta un feedback sobre el efecto de las píldoras, para ello, se anima el profesorado a crear un post cada vez que se utilice, cite o mencione una de las breves creaciones audiovisuales; asimismo se anima el alumnado a crear resúmenes de sus análisis de experiencias educomunicativas y sucesivamente a compartirlos.

Por lo tanto, todas estas propuestas se tendrán en cuenta para el planteamiento y desarrollo de la asignatura de los años sucesivos donde además se incorporará la practica de realizar más videoconferencias de las que se han realizado a lo largo de la asignatura.

\subsection{Reciclaje de la experiencia formativa}

Debido al planteamiento tan horizontal que ha tenido la asignatura de Didáctica seria incongruente no aportar la auto-evaluación que el mismo equipo docente ha realizado sobre la experiencia. Es evidente que cada año el grupo de alumnos variará por respuestas, pro-actividad, iniciativa, espíritu critico, sin embargo hay unos cuantos puntos que sería muy oportuno modificar debido a que son líneas muy generales de la metodología empleada. Indicamos a continuación algunos:

- El análisis educomunicativo de las experiencias debería de actualizarse para juntar en ello un análisis que incluya modelos no educomunicativos o que no se consideren exitosos; esto llevará a tener una visión aún más critica sobre los hechos y a poder comparar pautas distintas de proyectos exitosos o menos de las cuales se podrán educir aprendizajes. 
- El tiempo de adaptación que necesitan los alumnos y alumnas para acostumbrarse a la novedad de un modelo de enseñanza-aprendizaje totalmente multidireccional es muy exigente y necesita espacios específicos.

- Es muy importante encontrar una formula docente que se adapte a todos los factores involucrados en la docencia y nos referimos a la tecnología que se ha a disposición, a los docentes y al alumnado.

En definitiva es preciso hacer hincapié en la ponderación de la motivación generada y auto estimulada por y con todos los miembros de la comunidad, en cuanto verdadera aliada del aprendizaje. Esta reunión de recursos de ánimo es tan imprescindible como difícil de forzar.

\section{Referencias bibliográficas}

\section{Libros}

SILVA, M. (2006)"¿Nuevas que....aplicadas a qué...? El relicario pedagógico de los medios y las nuevas tecnologías"(pp 559-576) Madrid: UNED Comunicación educativa en la sociedad de la información

BOURDIEU, P.; PASSERON, J.C. (1979): La reproducción. Barcelona: Laia.

DÍAZ DE RADA, A.; VELASCO, H. (1996). La lógica de la investigación etnográfica. Madrid: Trotta.

FREIRE, P. (1970): Pedagogía del oprimido. Buenos Aires: Siglo XXI

(1997): La educación como práctica de la libertad. Madrid: Siglo XXI. (2003): El grito manso. Buenos Aires: Siglo XXI.

GENZUK, M. (2003): A synthesis of ethnographic research. University of Southern California Center for Multilingual, Multicultural Research.

KAPLÚN, M. (1998): Una pedagogía de la comunicación. Madrid: Ed. de la Torre. OSUNA ACEDO, S. (2007): Configuración y gestión de plataformas digitales. Madrid: UNED.

Capítulos o artículos en libros o revistas en papel

GIL FLORES, J. (1992-93). "La metodología de investigación mediante grupos de discusión". En Enseñanza \& Teaching: Revista interuniversitaria de didáctica, $\mathrm{n}^{\circ}$ 10-11. pp 199-214.

KAPLÚN, M. (1997). "De medio y fines en comunicación" en Chasqui, Revista latinoamericana de comunicación. $\mathrm{N}^{\circ}$ 58, Junio de 1997.

MATURANA, H. (2002). "Autopoiesis, Structural Coupling and Cognition: a history of these and other notions in the biology of cognition". En Cybernetics \& Human Knowing, Vol.9, No.3-4, pp. 5-34 
Artículos en publicaciones web:

ADICHIE, C. (2009): "The danger of a single story". En Oxford TED. Disponible subtitulado en http://www.caribbeanchoice.com/culture/content.asp?article=1645 [05-02-2011]

ANTÓN CUADRADO, R. (2010): "La web es un ser vivo". En comunicación extendida. Disponible en http://www.comunicacionextendida.com/la-web-es-un-ser-vivo/ [16-09-2013]

ARDEVOL. E. (2010): "El relato etnográfico". En Mediaciones, Antropología de los media. Disponible en http://eardevol.wordpress.com/2010/02/16/el-relato-etnografico/ [08-07-2012]

DOWNES, S. (2005): An Introduction to Connective Knowledge. Disponible en http://www.downes.ca/post/33034 [06-09-2013]

LÉVY, P. (2004): Inteligencia colectiva: por una antropología del ciberespacio. Disponible en: http://inteligenciacolectiva.bvsalud.org/public/documents/pdf/es/inteligenciaColectiva.pdf [14-08-2013].

SIEMENS, G. (2004): Una teoría de aprendizaje para la era digital. Disponible en: http://es.scribd.com/doc/26501532/Conectivismo-Siemens-Espanhol [08-09-2013]

\section{Los autores}

Raúl Antón Cuadrado, Campos de Interés: Creatividad conectada. Didáctica de la educación mediática. Colaborador académico del máster de educación y comunicación en la red (UNED). Master Geek en comunicacionextendida.com y eseeusee. com. Licenciado en Informática (UPM) Licenciado en Antropología Social y Cultural (UNED) Máster en educación y comunicación en la red (UNED)

Valeria Levratto, Máster en Comunicación y Educación en la Red y docente colaboradora en la UNED en la Facultad de Educación; Licenciada en Ciencias de la Comunicación, por la Facoltá di Lettere di Torino, Italia. Doctoranda en la UNED investiga las formas de lecturas en entornos digitales; a la vez participa en proyectos de Investigación sobre la Educomunicación y la Narrativa Digital. 